\title{
Special issue on two-dimensional materials
}

\author{
William Vandenberghe ${ }^{1} \cdot$ Vihar Georgiev ${ }^{2}$
}

Accepted: 27 January 2021 / Published online: 8 February 2021

(C) The Author(s), under exclusive licence to Springer Science+Business Media, LLC part of Springer Nature 2021

Ever since the discovery that graphite can be exfoliated down to a monolayer, a vast amount of research has been undertaken studying two-dimensional (2D) materials experimentally and theoretically. The first 2D material of interest was graphene, but in the meanwhile a host of other materials have been identified that can manifest themselves as 2D materials. A lot of research has been conducted toward transition-metal dichalcogenides like $\mathrm{MoS}_{2}$ or $\mathrm{WSe}_{2}$. Other materials of interest are transition-metal monochalcogenides like InSe or even black phosphorus has been realized in its monolayer form as phosphorene. As one of the main applications envisioned for 2D materials are electronic devices, 2D materials are a topic of major interest to the electronics community.

The computational study of 2D materials is a very important task to identify the intrinsic promise of 2D materials and to help understand unique experimental phenomena that are observed. The modeling of 2D materials has benefited extensively from the progress in first principles density functional theory, making it possible to calculate electronic and structural properties before a material is synthesized or characterized. At the same time, the modeling of 2D materials is pushing the boundaries of existing modeling methodologies previously developed for 3D materials.

In this special issue, we highlight some recent progress in the computational modeling of $2 \mathrm{D}$ materials.

The "Computational Electronics of Two-Dimensional Materials" Special Issue consists of 6 invited and 4 contributed papers giving an overview of computational electronics results and challenges related to two-dimensional (2D)

William Vandenberghe

william.vandenberghe@utdallas.edu

Vihar Georgiev

Vihar.Georgiev@glasgow.ac.uk

1 Department of Material Science and Engineering (MSE), University of Texas at Dallas, $800 \mathrm{~W}$ Campbell Rd, Richardson, TX 75080, USA

2 School of Engineering, University of Glasgow, Rankine Building (307B), Glasgow G12 8LT, Scotland, UK materials. The issue starts off with a review paper by Arnab K. Majee (University of Massachusetts-Amherst) describing recent progress on the modeling of the electrical and electrothermal properties of few-layered 2D devices. Next, a first principles study of the Electronic and Optical properties of WSe2/Cd0.9Zn0.1Te is reported by Anurag Chauhan et al. (Delhi Technological University). There are two papers dealing with carbon-based electronics: a first by Shanmeng Chen et al. (University of Texas at Dallas) investigating the impact of defects on graphene-nanoribbon field-effect transistors using a quantum transport approach and a second from Sadegh Biabanifard (Iran Analog Research Group) describing a THz absorber based on graphene patterns. Michel Houssa et al. deal with the issue of ferromagnetism in 2D materials and describe Doping-induced ferromagnetism in InSe and $\mathrm{SnO}$ monolayers. There are two contributions from Gautam Gaddemane et al. (University of Texas at Dallas). Gaddemane et al. reveal some important limitations of state-of-theart ab initio methods to predict the electronic transport of 2D materials from first principles in their first contribution, in their second contribution Gaddemane et al. perform a Monte Carlo analysis of phosphorene nanotransistors. Hosseini Sayed et al. discuss new resonant tunneling diodes based on graphene-black phosphorus heterostructures. Prasantha Mudimela et al. (Lovely Professional University) describe graphene-cantilever based digital logic gates. Finally, Jianwei Zhao et al. (Jiaxing University) study the effect of edge functionalization of monolayer $\mathrm{SiGe}$ nanoribbon transistors.

Publisher's Note Springer Nature remains neutral with regard to jurisdictional claims in published maps and institutional affiliations. 